\title{
Randomized Controlled Trial of a Breath-Actuated Nebulizer in Pediatric Asthma Patients in the Emergency Department
}

\author{
Katie Sabato MSc RRT, Priscilla Ward RRT, William Hawk MD, \\ Virginia Gildengorin PhD, and Jeanette M Asselin RRT MSc
}

\begin{abstract}
BACKGROUND: Bronchodilator treatment for asthma can be provided with various aerosolgenerating devices and methods. There have been no randomized trials of a breath-actuated nebulizer versus continuous 1-hour nebulization and/or small-volume constant-output nebulizer in pediatric asthma patients. METHODS: We conducted a randomized study of one-time albuterol treatment with the AeroEclipse breath-actuated nebulizer versus standard therapy (single treatment via small-volume nebulizer or 1-hour of continuous nebulized albuterol) in pediatric asthma patients in the emergency department. Eligible patients were those admitted to the emergency department, 0 months to 18 years of age, who presented with asthma or wheezing. We assessed all the patients with our clinical asthma scoring system and peak-flow measurement if possible. We stratified the patients by clinical asthma score and weight, and then randomized them to receive their initial albuterol treatment in the emergency department via either AeroEclipse or standard therapy. We recorded time in the emergency department, change in clinical asthma score, need for additional bronchodilator treatments, need for admission, patient response, ability to actuate the AeroEclipse, and adverse effects. RESULTS: We enrolled 149 patients between October 14, 2004 and November 11, 2005, and we randomized 84 patients to AeroEclipse and 65 to standard therapy. The cohort's average age was 5.5 years. There were no significant differences in demographics. The initial mean clinical asthma scores were $5.1 \pm 2.4$ in the AeroEclipse group, and $5.1 \pm 2.1$ in the standard-therapy group. Time in the emergency department was not different (AeroEclipse $102 \mathrm{~min}$, standard therapy $125 \mathrm{~min}, P=.10$ ), but the AeroEclipse group had a significantly greater improvement in clinical asthma score $(1.9 \pm 1.2$ vs $1.2 \pm 1.4, P=.001)$ and respiratory rate $(P=.002)$, and significantly lower admission rate $(38 \%$ vs $57 \%, P=.03)$. There was no difference in adverse effects. CONCLUSIONS: Although AeroEclipse did not reduce the time in the ED, it significantly improved clinical asthma score, decreased admissions, and decreased respiratory rate. Key words: asthma; bronchodilator; aerosol; nebulizer; breath-actuated; pediatric; albuterol; emergency; department; clinical asthma score. [Respir Care 2011;56(6):761-770. () 2011 Daedalus Enterprises]
\end{abstract}

\section{Introduction}

Asthma is the most common chronic illness of childhood. In the emergency department (ED) at Children's

\footnotetext{
Ms Sabato and Ms Ward are affiliated with the Department of Respiratory Care; Dr Hawk is affiliated with the Emergency Department; Dr Gildengorin is affiliated with the Clinical and Translational Science Institute; and Ms Asselin is affiliated with the Department of NeonatalPediatric Research, Children's Hospital and Research Center Oakland, Oakland, California.
}

Ms Sabato presented a version of this paper at the 51st International Respiratory Congress of the American Association for Respiratory Care, held December 3-6, 2005, in San Antonio, Texas.
Hospital and Research Center Oakland, 5,498 patients were treated for asthma in 2005 . The $\beta_{2}$ agonist albuterol is the standard first-line aerosol therapy in the treatment of children with asthma. Effective, efficient, and safe delivery of albuterol affect the amount of time in the ED and whether

\footnotetext{
The authors have disclosed no conflicts of interest.

Correspondence: Katie Sabato MSc RRT, Department of Respiratory Care, Children's Hospital and Research Center Oakland, 747 52nd Street, Oakland CA 94609. E-mail: ksabato@mail.cho.org.
}

DOI: $10.4187 /$ respcare. 00142 


\section{Breath-Actuated Nebulizer in Pediatric Asthma}

hospital admission is required. Factors associated with effective aerosol delivery are: choice of nebulizer, aerosol particle size, nebulizer/patient interface, patient size, patient adherence to therapy, dose, and degree of bronchoconstriction (asthma severity). Infants have rapid respiratory rate, nose breathing, little cooperation with therapy, and other obstacles to successful aerosol delivery. ${ }^{1-4}$

\section{See the Related Editorial on Page 882}

Albuterol treatments for asthma can be provided via various aerosol generating devices and methods, which can be institution-specific and are widely variable. ${ }^{5}$ Smallvolume constant-output jet nebulizers (SVNs) are very popular in pediatric EDs. Most SVNs generate aerosol constantly, during both inhalation and exhalation, and typical SVN treatments require 8-15 min, and, often, repeat treatment. Approximately 14 years ago, some manufacturers introduced large-volume nebulizers capable of delivering albuterol over a longer period: generally $1-8$ hours. In theory, this longer-period delivery eliminates the peaks and troughs of intermittent SVN treatments and decreases the need for the respiratory therapist (RT) to return to the patient bedside. The available research on continuous nebulization, although limited, suggests they are safe and effective. ${ }^{6-8}$ Many pediatric institutions have incorporated continuous nebulization into their asthma regimens. We use both SVN and continuous nebulization in our ED asthma algorithm.

Aerosol particle size and nebulizer type affect aerosol delivery. Choosing a nebulizer requires close attention to the device's ability to deliver a high percentage of particles in the respirable range $(2-5 \mu \mathrm{m}),{ }^{9-11}$ which is the range recognized in many standards relating to aerosol delivery. Particles $<2 \mu \mathrm{m}$ can reach the lungs but are likely to be exhaled instead of deposited in the lungs, particularly if $<1 \mu \mathrm{m}$. Traditional SVNs deliver some particles in the $<5 \mu \mathrm{m}$ range, but their output is extremely variable, ${ }^{12,13}$ and they lose respirable particles in the continuous flow during exhalation. Some newer nebulizers can deliver a higher percentage of respirable-range particles, ${ }^{14}$ and some newer nebulizers are breath-actuated (ie, aerosol production is started by the onset of inhalation) to limit the loss of aerosol during exhalation. The AeroEclipse (Monaghan Medical, Plattsburgh, New York) is a breathactuated SVN, introduced in January 2000. The AeroEclipse can also be manually actuated, for patients too weak or too young to trigger the flow valve, and can be set to nebulize continuously by placing a cap over the manual button. Studies have shown good aerosol delivery and efficacy with AeroEclipse, ${ }^{15-18}$ but there are no large pediatric trials of AeroEclipse.
Nebulizer effectiveness is also influenced by the patient interface (eg, mouthpiece, mask, blow-by), particularly in children. Young children have limited ability to use a mouthpiece or mask as the aerosol interface. Children do not react favorably to devices or aerosol directed at the face. With younger pediatric patients, RTs often have to resort to blow-by treatment. With mask or blow-by, children often have a reflex "pull-away" response, with distress and/or crying. Iles et al found negligible aerosol deposition in crying and distressed children. ${ }^{3}$

There appears to be no consensus regarding the optimal albuterol dose or dilution for a child. Some authors have suggested that adult doses of aerosolized bronchodilator are comparable when used in children. ${ }^{19}$ Crain et al noted that most dosing in pediatric hospitals is institution-specific. 5 Some pediatric institutions, including ours, have developed their own approaches to bronchodilator therapy. In 1984, Kao et al studied full-strength isoproterenol hydrochloride for the treatment of bronchoconstriction in neonates. ${ }^{20}$ The concentrated solution was nebulized and administered for a specific number of "breaths," with good results. Kao joined our staff in 1985, and her work initiated our change to undiluted aerosol therapy that year.

Gutglass et al evaluated undiluted albuterol via SVN in children in the ED and found that undiluted albuterol was as safe and effective as diluted albuterol. ${ }^{21}$ Our current standard SVN nebulizer is the Misty-Neb (Cardinal Healthcare, Dublin, Ohio), and our albuterol dosage is $0.5 \mathrm{~mL}$ of albuterol in $0.5 \mathrm{~mL}$ of normal saline to infants $\leq 20 \mathrm{~kg}$, and $1 \mathrm{~mL}$ of undiluted albuterol to patients $>20 \mathrm{~kg}$. With the introduction of 1-8-hour continuous nebulization, ${ }^{6-8}$ patients presenting with more severe exacerbation receive $10 \mathrm{mg}$ aerosolized albuterol over 1 hour, via the high-flow MiniHeart nebulizer (WestMed, Tucson, Arizona).

In 1994, to standardize asthma treatment, we created an algorithm (Fig. 1) that uses our modification (Table 1) of the clinical asthma scoring system validated by Schuh et al. ${ }^{22}$ Patients are scored from 0 to 3 , on accessory muscle use, breath sounds, and dyspnea; each attribute has equal weight. Patients $<2$ years old cannot be scored on dyspnea. Two additional points can be assigned if PEF is $<70 \%$ of predicted (one point) or oxygen saturation is $<92 \%$ on room air (one point).

The algorithm dictates that children with a low clinical asthma score (milder asthma symptoms) receive albuterol via constant-flow SVN. The dose is determined by patient weight, as described above. Children with a high clinical asthma score (more severe asthma symptoms) receive $10 \mathrm{mg}$ albuterol via continuous nebulization, over 1 hour. This very aggressive approach to asthma care has worked well at our center. In 2002 we assessed the validity and reliability of our clinical asthma score system, and presented our findings at the Open Forum of the International Respiratory Congress of the American Association for Re- 


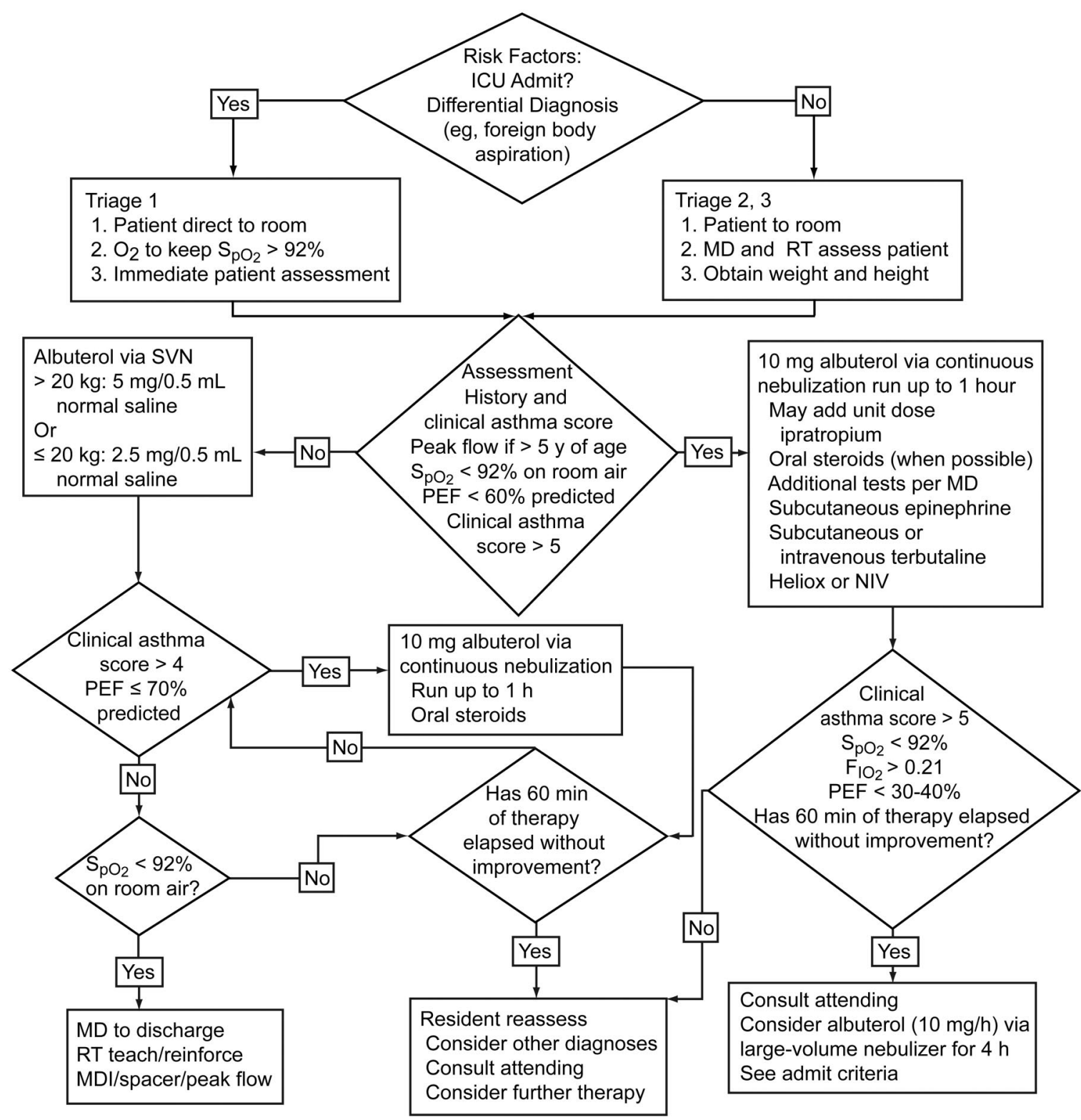

Fig. 1. Emergency department asthma algorithm. ICU = intensive care unit. MD = physician. RT = respiratory therapist. SVN $=$ smallvolume nebulizer. PEF = peak expiratory flow. NIV = noninvasive ventilation. $\mathrm{MDI}=$ metered-dose inhaler.

spiratory Care. ${ }^{23}$ Since then the clinical asthma score has been part of our asthma assessment. RTs carry a clinical asthma score reference card with their name badge, as a constant reminder of the clinical asthma scoring system. New employees undergo formal training in the asthma scoring system during orientation. Our staff choose the interface (mask, mouthpiece, or blow-by) based on the patient's age, coordination, and asthma severity.

Comparisons of continuous nebulization and SVN have been limited ${ }^{8,22-26}$ but have shown continuous nebulization to be as safe and effective as SVN. There have been no randomized controlled trials of AeroEclipse versus standard aerosol delivery in pediatric patients in the ED. We compared one-time treatment with AeroEclipse to our 2 current standard treatments (SVN and continuous nebulization) in the ED.

\section{Methods}

The study protocol was approved by our institutional review board and ED physicians. The study was partly funded by Monaghan Medical, which provided a grant and the AeroEclipse nebulizers used in the study. However, Monaghan Medical had no input into the study design, data analysis, or manuscript review. Prior to the study we 


\section{Breath-Actuated Nebulizer in Pediatric Asthma}

Table 1. Clinical Asthma Score System

\begin{tabular}{|c|c|c|c|}
\hline $\begin{array}{c}\text { Clinical } \\
\text { Asthma Score* }\end{array}$ & Accessory Muscle Use & Breath Sounds/Wheezing & Dyspnea \\
\hline 0 & No retractions & No wheezing & No dyspnea \\
\hline 1 & Intercostal retractions & End-expiratory wheezing & Some dyspnea, but normal activity and speech \\
\hline 2 & $\begin{array}{l}\text { Intercostal and/or substernal } \\
\text { retractions }\end{array}$ & Inspiratory and expiratory wheezing & $\begin{array}{l}\text { Moderate dyspnea: decreased activity, 5-8-word } \\
\text { sentences }\end{array}$ \\
\hline 3 & $\begin{array}{l}\text { Nasal flaring, with or without } \\
\text { retractions }\end{array}$ & $\begin{array}{l}\text { Wheezing audible without stethoscope, } \\
\text { or chest is silent }\end{array}$ & $\begin{array}{l}\text { Severe dyspnea: concentrates on breathing, would } \\
\text { rather not speak }\end{array}$ \\
\hline
\end{tabular}

* Add 1 for peak flow $<70 \%$ of predicted. Add 1 for $\mathrm{S}_{\mathrm{pO}_{2}}<92 \%$ on room air

held in-services to familiarize the respiratory and emergency department staff with the study. To limit variability in execution of the study protocol, we limited patient enrollment to the day shift (7:00 AM to 7:00 PM) and a small number of respiratory care staff sought the patient consents and enrolled patients. Four RTs completed training in protection of human subjects' rights, closely reviewed the study eligibility and protocol, obtained the consents, and performed the study procedures. These study staff were well versed with the clinical asthma score and our asthma algorithm, which are part of our routine care.

Children admitted to the ED between birth and 18 years of age, with breathing difficulties associated with wheezing or asthma, were eligible for enrollment. We excluded patients who had a history of structural heart disease; bronchiolitis and were $<2$ years old; or were ordered to receive racemic epinephrine aerosol.

After initial nursing assessment in triage, we approached the patient's parents to ask about study participation. If parental consent was obtained, the patient was examined by one of the study respiratory staff and assessed for baseline clinical asthma score. Patients were then stratified by weight $(\leq 20 \mathrm{~kg}$ or $>20 \mathrm{~kg}$ ) and clinical asthma score $(\leq 4$ or $>4)$, and randomized within each strata, with a randomization table (4-group block design, created a priori), to receive their initial bronchodilator treatment via either standard therapy (continuous-output SVN or continuous nebulization) or AeroEclipse (Fig. 2). Standard therapy was guided by our asthma algorithm and the discretion of the attending physician. Standard-therapy patients with clinical asthma score $>4$ received continuous nebulization. Patients randomized to AeroEclipse received albuterol via AeroEclipse regardless of clinical asthma score. The decision to admit a patient to the hospital was determined by the attending physician.

Bronchodilator dose was based on patient weight and the nebulizer used. The AeroEclipse and SVN patients $\leq 20 \mathrm{~kg}$ received $0.5 \mathrm{~mL}(2.5 \mathrm{mg})$ albuterol in $0.5 \mathrm{~mL}$ normal saline. AeroEclipse and SVN patients $>20 \mathrm{~kg}$ received $1.0 \mathrm{~mL}(5 \mathrm{mg})$ albuterol undiluted. Continuous nebulization patients received $2.0 \mathrm{~mL}(10 \mathrm{mg})$ albuterol in
$18 \mathrm{~mL}$ normal saline (ie, $20 \mathrm{~mL}$ nebulizer charge) over 1 hour.

If the patient was able to perform a peak expiratory flow (PEF) maneuver, we attempted to obtain PEF measurements (Truzone, Monaghan Medical, Plattsburgh, New York) before and after treatment.

Mouthpiece was the preferred interface with AeroEclipse and SVN. If the patient did not have the coordination to use a mouthpiece, we attempted to use a mask. SVN patients unable to use a mouthpiece and all continuous nebulization patients used conventional pediatric or adult aerosol masks. AeroEclipse patients who required a mask used the Comfort Seal mask (Monaghan Medical, Plattsburgh, New York). We assessed patient tolerance of all masks used, during the first 10 breaths of treatment. If patient did not tolerate the mask, we quickly removed it and aerosol was given via blow-by, as close as possible to (without touching) the nose and mouth.

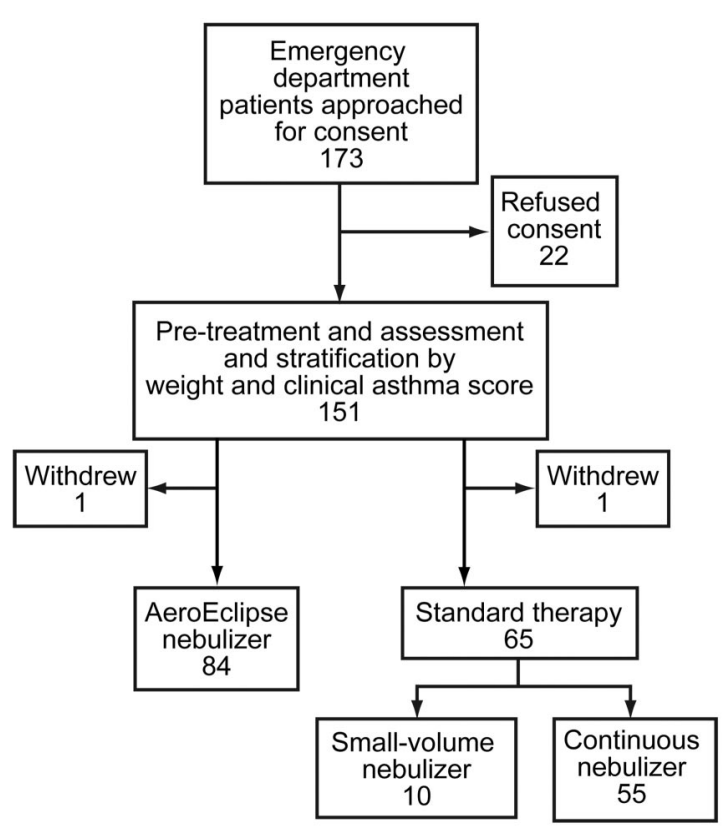

Fig. 2. Flow chart. 
Patients randomized to AeroEclipse and able to breathactuate it were allowed to do so regardless of age. With patients who were unable to breath-actuate the AeroEclipse within the first 10 breaths, we set the AeroEclipse in continuous-nebulization mode.

Demographic data were recorded on all patients. All patients, regardless of randomization assignment, were assessed prior to and 10 min after completion of their assigned treatment. Although times between before and after assessments differed in minutes, patients were consistently assessed 10 min after completion of assigned drug dose delivery. Assessments included clinical asthma score, heart rate, and respiratory rate. We recorded PEF in patients who could complete the PEF maneuver. In addition, level of nausea, incidence of tremors, vomiting, hyperactivity, need for additional treatments, steroids, time in the ED, and hospital admission were recorded. Length of stay in the ED was defined as the time patient treatment was initiated to the time orders were written to admit patient or send them home.

We recorded patient tolerance of the aerosol treatments as either: tolerated treatment without problems; tolerated treatment with encouragement; some resistance to treatment; or total resistance to treatment and required blowby. We also recorded ability to breath-actuate the AeroEclipse.

\section{Statistical Analysis}

Prior to the study we conducted a power analysis to determine the sample size. Based on previously collected data on respiratory care treatments and time in the ED, the estimated standard deviation for time in the ED was 1.44 hours for patients not admitted, and 1.68 hours for patients admitted. We calculated that a sample size of 150 (75 patients per group) would have $90 \%$ power to detect a difference of $\geq 55$ min between the groups.

We analyzed time in the ED with the Student $t$ test. We used analysis of covariance to analyze the changes in clinical asthma score. Heart rate, respiratory rate, and PEF were analyzed with a 2-sample $t$ test to compare treatment groups. In patients who attempted PEF, if the result was zero, that value was changed to one for analysis. For dichotomous outcomes (yes/no, admission, ED disposition, and complications) we used chi-square or the Fisher exact test.

We conducted additional analyses of time in the ED, ED disposition, and clinical asthma score to evaluate the effect of treatment type (continuous nebulization or SVN) in the standard-therapy group, compared to the AeroEclipse group. For these analyses we used analysis of variance for 3 groups, and if we found a significant difference between the 3 groups, we used the Tukey-Kramer method of multiple comparisons to determine which group differed.
Data analysis was with statistics software (SAS 9.2, SAS Institute, Cary, North Carolina). A significance level of .05 was used for all statistical tests.

\section{Results}

Between October 14, 2004, and November 11, 2005, approximately 4,000 children were admitted to our emergency department with breathing difficulties associated with asthma or wheezing. One-hundred fifty-one children consented, and 2 withdrew consent prior to treatment, and 149 completed therapy and were analyzed. Twenty-two families refused consent, primarily due to concerns over possible delay in symptom recovery with a research treatment.

Eighty-four patients were randomized to AeroEclipse, and 65 to standard therapy (55 to continuous nebulization, 10 to SVN). Demographics at baseline showed no difference between the study groups (Table 2). The groups were comparable in both age and weight. The initial mean clinical asthma scores were $5.1 \pm 2.4$ in the AeroEclipse group, and $5.1 \pm 2.1$ in the standard-therapy group. The clinical asthma score range was $0-10$ in the AeroEclipse group, and $0-11$ in the standard-therapy group.

The average time in the ED was less in the AeroEclipse group, but this did not reach statistical significance (AeroEclipse $102 \pm 77 \mathrm{~min}$, standard-therapy group $125 \pm 88 \mathrm{~min}, P=.10$ ). The AeroEclipse group had better improvement in clinical asthma score than the standardtherapy group: $38 \%$ of the AeroEclipse patients versus $24 \%$ of the standard-therapy patients had an improvement in clinical asthma score $(P=.01)$. While the groups' baseline clinical asthma scores were similar, the mean improvement in clinical asthma score after treatment was greater in the AeroEclipse group $(1.9 \pm 1.2)$ than in the standard-therapy group $(1.2 \pm 1.4)(P=.001)$ (Fig. 3). Change in heart rate and $\mathrm{S}_{\mathrm{pO}_{2}}$ were not different between the groups. However, there was an unexpected, significant drop in respiratory rate in the AeroEclipse group $(P=.002)$ (Fig. 4). Only a few patients were able to perform the PEF maneuver (AeroEclipse 28 [33\%], standard therapy 20 [31\%], difference not significant).

The incidence of clinical adverse effects (cough, nausea, vomiting, tremors, and/or hyperactivity) was not different between the groups, before or after treatment (Fig. 5). There was no difference in the need for other therapies, such as additional bronchodilator (AeroEclipse 58 [69\%], standard therapy 37 [57\%], $P=.17$ ) or number of patients who received steroids (AeroEclipse 66 [79\%], standard therapy 46 [71\%], $P=.34)$. However, fewer AeroEclipse patients required hospital admission (AeroEclipse 39 [40\%], standard therapy 40 [62\%], $P=.03$ ).

We performed a secondary analysis of AeroEclipse versus the 55 continuous nebulization therapies and $10 \mathrm{SVN}$ therapies for time in the ED, ED disposition, and change in 
Table 2. Patient Demographics at Entry $(\mathrm{n}=149)$

\begin{tabular}{lccc}
\hline \hline & AeroEclipse & Standard Therapy & $P$ \\
\hline Male/female (no.) & $48 / 36$ & $44 / 21$ & .13 \\
Age (y) & $5.7 \pm 4.8$ & $5.4 \pm 4.4$ & .68 \\
Weight (kg) & $28.4 \pm 24.5$ & $28.3 \pm 24.3$ & .98 \\
Weight $<$ 20 kg (no.) & 41 & 35 & .62 \\
Weight $\geq 20 \mathrm{~kg}$ (no.) & 43 & 30 & .62 \\
Heart rate (beats/min) & $129 \pm 29$ & $127 \pm 28$ & $.82 \pm 15$ \\
Respiratory rate (breaths/min) & $37 \pm 16$ & $5.1 \pm 2.1$ & .75 \\
Initial clinical asthma score & $5.1 \pm 2.4$ & 23 & .13 \\
Clinical asthma score 0-4 (no.) & 36 & $2.9 \pm 1.1$ & 42 \\
Clinical asthma score 0-4 & $2.8 \pm 1.2$ & $6.3 \pm 1.4$ \\
Clinical asthma score $>4$ (no.) & 48 & & .30 \\
Clinical asthma score $>4$ & $6.8 \pm 1.5$ & & .36 \\
& & & .30 \\
\pm values are mean \pm SD. & & & \\
\hline
\end{tabular}

clinical asthma score, to see if there was a differential effect by standard-therapy treatment assignment. We evaluated the baseline status of the standard-therapy group by treatment (continuous nebulization or SVN). As expected, given our asthma algorithm, the continuous nebulization patients had a higher average clinical asthma score (5.5) than the SVN patients (3.0). There was no significant difference in baseline clinical asthma score between the AeroEclipse group $(5.1 \pm 2.4)$ and the continuous nebulization group $(5.5 \pm 1.9)(P=.58)$, but the SVN group's baseline score was significantly lower $(3.0 \pm 2.1)$ $(P=.02)$.

The AeroEclipse group had significantly better improvement in clinical asthma score than did the continuous nebulization group: $-1.7 \pm 1.5$ vs $-1.1 \pm 1.4$ $(P=.018)$. Neither continuous nebulization nor AeroEclipse showed an improvement in clinical asthma

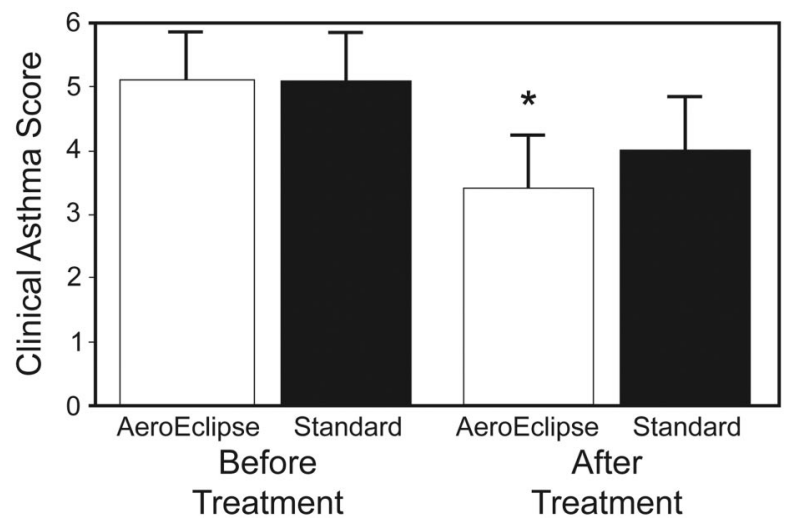

Fig. 3. Clinical asthma score before and after nebulized albuterol via either AeroEclipse breath-actuated nebulizer or standard therapy (regular small-volume nebulizer or continuous nebulization). The data bars represent mean values, and the error bars represent standard deviations. ${ }^{*} P=.001$. score over SVN, possibly due to the small number of SVN patients $(n=10)$.

Time in the ED was nonsignificantly longer in the continuous nebulization patients: AeroEclipse $102 \pm 77 \mathrm{~min}$, continuous nebulization $137 \pm 89 \mathrm{~min}, \mathrm{SVN} 45 \pm 33 \mathrm{~min}$ $(P=.10)$. Three patients were removed from this analysis because they were discovered to be direct admits (they were going to be admitted regardless of therapy). The continuous nebulization patients had the highest admission rate: 36 continuous nebulization patients $(65 \%), 34$ AeroEclipse patients $(40 \%), 4 \mathrm{SVN}$ patients $(40 \%)(P=.059)$.

Bigger (older) patients $(>20 \mathrm{~kg})$ were more likely to have improvement with AeroEclipse than were patients $\leq 20 \mathrm{~kg}$ (change in clinical asthma score with AeroEclipse -2.1 vs standard therapy $-1.2, P=.007$ ) (Fig. 6). The patients with baseline clinical asthma score $>4$ were more likely to have greater response with

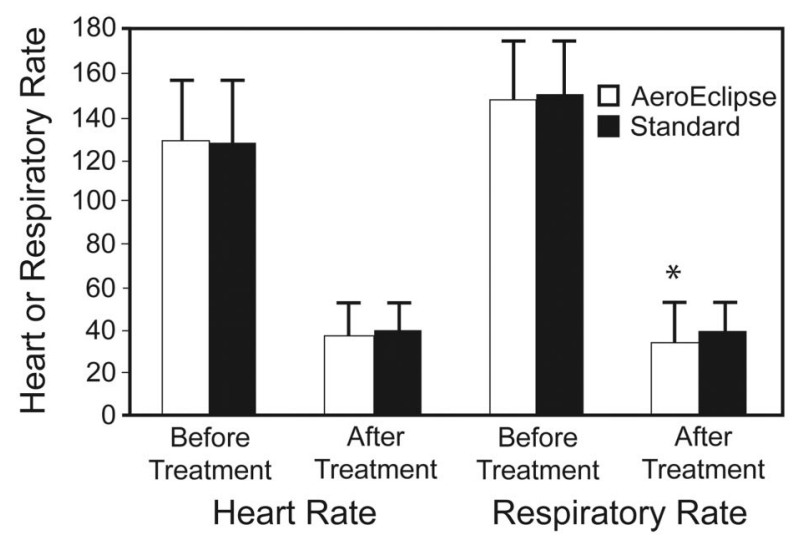

Fig. 4. Change in heart rate and respiratory rate before and after nebulized albuterol via either AeroEclipse breath-actuated nebulizer or standard therapy (regular small-volume nebulizer or continuous nebulization. The data bars represent mean values, and the error bars represent standard deviations. ${ }^{*} P=.002$. 


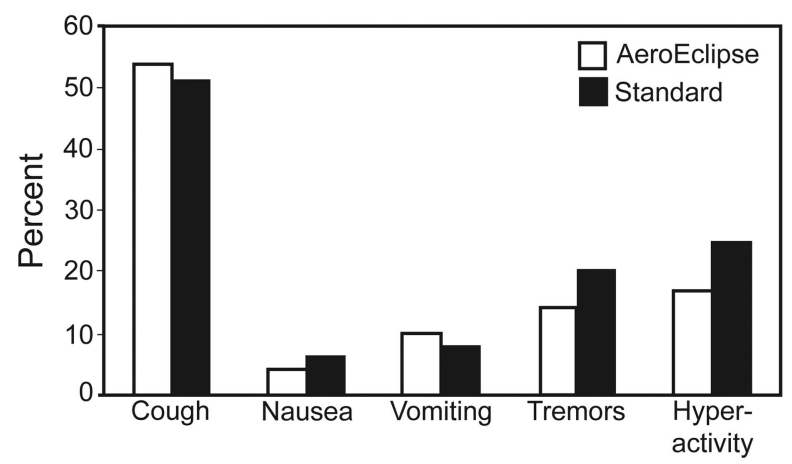

Fig. 5. Adverse effects after nebulized albuterol via either AeroEclipse breath-actuated nebulizer or standard therapy (regular small-volume nebulizer or continuous nebulization). There were no significant differences.

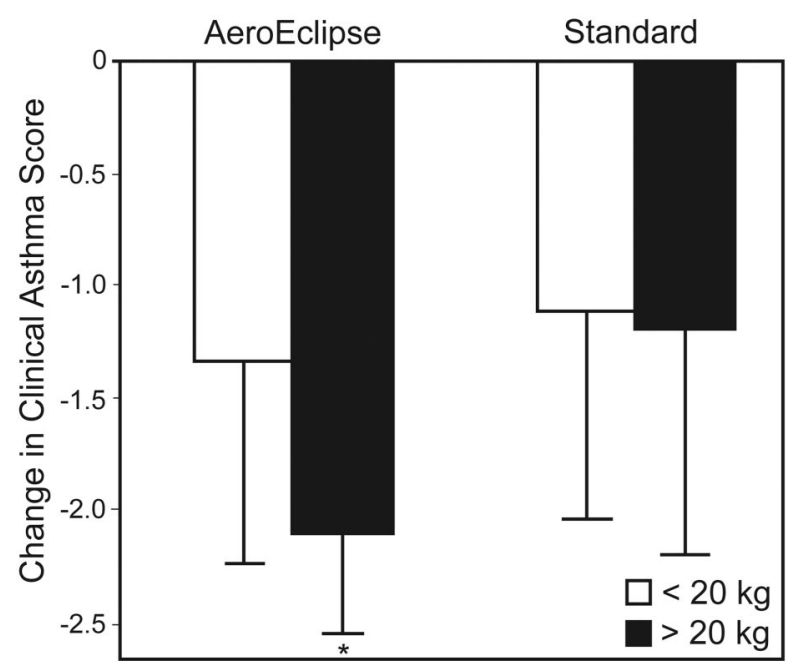

Fig. 6. Change in clinical asthma score versus patient-weight category. Patients $>20 \mathrm{~kg}$ had greater response with AeroEclipse than did patients $\leq 20 \mathrm{~kg}$. The data bars represent mean values, and the error bars represent standard deviations. ${ }^{*} P=.007$.

AeroEclipse than were those with baseline score $\leq 4$ (change in clinical asthma score with AeroEclipse -2.2 vs standard therapy $-1.5, P=.01$ ) (Fig. 7).

Treatment tolerance was collapsed into 2 groups: accepted treatment or resisted treatment. Seventy-three AeroEclipse patients (87\%) versus 48 standard-therapy patients $(74 \%)$ accepted their aerosol treatment outright $(P=.06)$. Of the patients who resisted treatment (AeroEclipse 9 [11\%], standard therapy 17 [65\%]), fewer AeroEclipse patients resisted to the point that they required blow-by (AeroEclipse 3/9 [33\%], standard therapy 15/17 [88\%], $P=.007$ ) (Fig. 8).

Although there has been some concern about pediatric patients' ability to breath-actuate, 66 (79\%) of the 84 AeroEclipse patients were able to breath-actuate, and their average age was 6.7 years (range 6 months to 18 years).

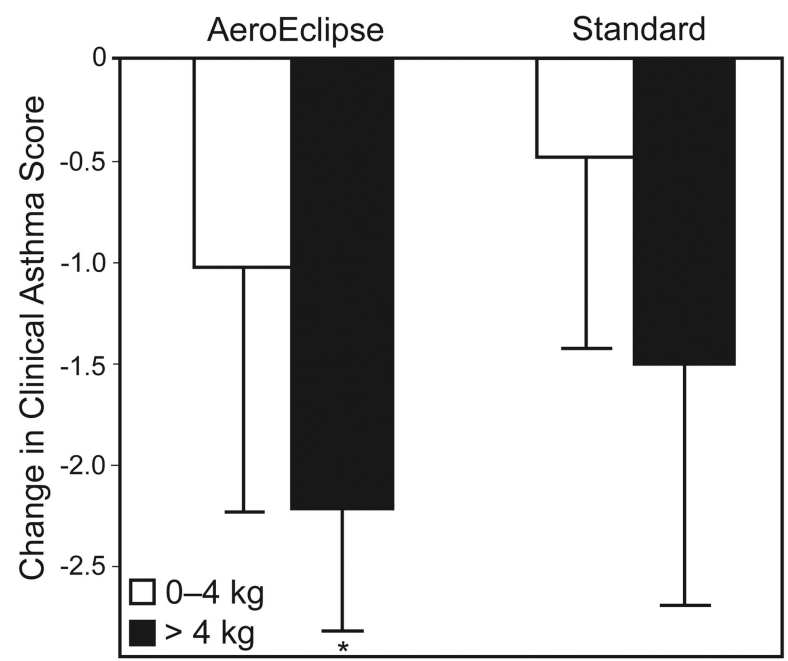

Fig. 7. Change in clinical asthma score versus baseline clinical asthma score category. Patient with baseline clinical asthma score $>4$ had significantly greater response with AeroEclipse than did patients with baseline clinical asthma score $\leq 4$. The data bars represent mean values, and the error bars represent standard deviations. ${ }^{*} P=.01$.

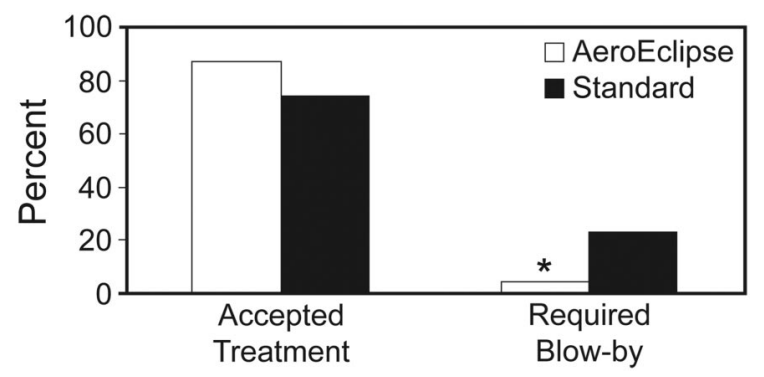

Fig. 8. Percent of patients who tolerated the aerosol treatment versus required blow-by. ${ }^{*} P=.007$.

Some patients with high clinical asthma score (severe asthma) were initially unable to breath-actuate, but after 4-6 breaths with the clinician manually actuating the AeroEclipse, these patients were able to breath-actuate for the remainder of the treatment.

\section{Discussion}

Our results show that albuterol delivery via AeroEclipse as the initial treatment for asthma patients in our ED is more effective than initial treatment with continuous nebulization. Although there was no difference in time in the $\mathrm{ED}$, the AeroEclipse patients had significantly better improvement in clinical asthma score, better decrease in respiratory rate, and a lower admission rate, and there was no significant difference in adverse effects.

Our study was powered to detect a difference in time in the ED, but we found no difference. Time in the ED is 
affected by many uncontrollable factors such as physician availability, bed availability, patient load, and social issues. In addition, in this study, comparison of time in the ED is confounded by the difference in duration of treatment determined by the clinical asthma score and treatment allocation. The nonsignificant difference in time in the ED may reflect these confounders. The decision to admit a patient was by the treating physician, and study staff had no role in this. Although the decision to admit was not standardized, fewer patients in the AeroEclipse group required hospital admission (39 [46\%] vs 40 [62\%], $P=.09$ ).

The average pre-treatment clinical asthma score was 5.1 in both groups, so these patients had moderate to severe asthma, which is also reflected by the number of standardtherapy patients who required continuous aerosol therapy (continuous nebulization) according to our asthma algorithm (see Fig 1). The AeroEclipse patients had significantly better improvement in clinical asthma score than the standard-treatment group as a whole; however, the secondary analysis revealed that this difference was limited to the continuous nebulization group. The observed difference in asthma score improvement and admission rate might be due to better nebulizer efficiency with AeroEclipse. There was no significant difference in asthma score improvement between the AeroEclipse group and the SVN group, but this finding may be due to the small number of SVN patients; however, this requires further study.

The patients $>20 \mathrm{~kg}$ had greater benefit from AeroEclipse, as did the sicker patients (baseline score $>4$ ). The better efficacy of AeroEclipse may be the result of breath actuation, smaller aerosol particle size, and/or greater patient acceptance. Breath-actuation results in a greater inhaled drug mass and less medication waste. ${ }^{13,16}$ Seventynine percent of the AeroEclipse patients were able to breathactuate. Continuous nebulization with regular SVN or continuous nebulization results in considerable loss of drug to the environment, and reduced delivery to the lung.

Nebulizer performance is based on output volume and particle size. The more particles in the respirable range and the greater the density of respirable-range particles, the more effective the aerosol delivery to the lower respiratory tract. ${ }^{14}$ Several studies have found that AeroEclipse delivers more respirable-range particles than some older-model nebulizers. ${ }^{15-18}$ On average, $80 \%$ of the aerosol from AeroEclipse is $<4.8 \mu \mathrm{m}$, and this small average particle size may improve lung deposition.

Some have argued that the choice of interface and interface design affect aerosol delivery. ${ }^{27}$ In a study with 359 children, ages 6 months to 8 years, with mild persistent asthma, Virant found that aerosol delivery was equally effective via mask or mouthpiece, ${ }^{28}$ and studies by Malone et al, ${ }^{29}$ Mellon et al, ${ }^{30}$ and Lowenthal and Kattan ${ }^{31}$ support that finding. Mask design and face seal (fit) may play a large role in lung deposition. The masks we used in our standard-therapy patients were those we traditionally use with continuous nebulization or SVN. They are vented, somewhat rigid, plastic masks that do not form fit to the child's face, and they have large areas from which aerosol can escape, which can increase aerosol deposition on the face and eyes and decrease delivery to the lung. ${ }^{32}$

Amirav and Newhouse highlighted the importance of a tight face-mask seal. ${ }^{33}$ They concluded that a well fitted mask can increase lung delivery by $30 \%$. They evaluated 3 masks and found that the mask that comes with the AeroChamber holding chamber and a mask made by Hans Rudolph Company provided the best seals. AeroEclipse uses that same AeroChamber mask, which is made of pliable silicone (and is thus more form fitting) and is nonvented. Those design features seem to facilitate patient comfort and acceptance. Patient tolerance of an aerosol treatment often relates to interface type, and can play a large role in determining effective drug delivery. In addition, better fitting masks and nebulization only during inspiration may help limit clinician exposure to aerosol particles, which can carry microbes.

Some of the patient resistance to aerosol therapy in children is their dislike/intolerance of anything blowing in their face. They commonly pull away and/or cry, resulting in wasted medication. Several studies have shown that aerosol deposition to a distressed, crying, or combative child is negligible. ${ }^{4,33,34}$ Most agree that blow-by is ineffective. ${ }^{34-36}$ Our AeroEclipse patients accepted their treatments more often than our standard-therapy patients, but this did not reach statistical significance (AeroEclipse 87\% vs standard therapy $74 \%, P=.06$ ). Although the patients numbers were small, fewer patients in the AeroEclipse group required blow-by (AeroEclipse 3 vs standard therapy $15, P=.004)$. The design of AeroEclipse and AeroChamber mask may have been a factor in the higher acceptance in the AeroEclipse group.

In this study one could argue that the dosages in the 3 groups differed. The AeroEclipse and SVN patients received $2.5-5 \mathrm{mg}$ of albuterol, either undiluted or only slightly diluted, over a short period, whereas the continuous nebulization patients received $10 \mathrm{mg}$ of albuterol over 1 hour. However, the SVN and continuous nebulization patients may have received less drug because, with regular SVN and continuous nebulization, nebulization is throughout the respiratory cycle, so some aerosol is lost to the environment. This could account for the better improvement in asthma score in the AeroEclipse group.

While this study did not directly address resource utilization, one could argue that the time taken to deliver a breath-actuated nebulizer treatment is much less than that to deliver a continuous nebulization treatment. Recently we did an ad hoc e-mail survey of respiratory managers of 


\section{Breath-Actuated Nebulizer in Pediatric Asthma}

the 42 children's hospitals in the Children's Health Consortium of America. Thirty-eight of the 42 managers responded and indicated that they use continuous nebulization in their EDs, and many of them answered "a lot!" (unpublished data). That survey result supports the relevance of this study, and our results may be important to children's hospitals nationwide. The significantly better improvement of asthma score in the AeroEclipse group has potential implications on resource utilization by respiratory care staff.

One potential weakness of our study relates to direct observation of the treatment administration. The RT always remained at the bedside during AeroEclipse and SVN treatments, but continuous nebulization patients were not continuously observed. As is normal practice, the continuous nebulization patients were initially assessed, set up on continuous nebulization, and then left under the supervision of the parent and ED nurse. The RT returned near the end of the continuous nebulization treatment to evaluate treatment response. Given reports by parents and ED staff, we assumed that the child remained on the continuous nebulization continuously during that hour.

There were no significant differences between AeroEclipse and standard-therapy in patient safety or adverse effects, and all remained within safe ranges. There was a significant drop in respiratory rate in the AeroEclipse group.

Continuous nebulization of aerosols throughout inspiration and exhalation may pose a risk to clinicians and family members because aerosol is released to the environment during exhalation. This problem can be reduced with the AeroEclipse nebulizer.

We conducted this study to see if a newer nebulizer technology, with purportedly higher efficiency and shorter treatment time, could replace our current SVNs and continuous nebulization treatments in our emergency department. We found that initial treatment with AeroEclipse was more efficacious than initial treatment with continuous nebulization, which we currently use with patients with more severe asthma. An important finding was that our sicker patients (baseline asthma score $>4)$ and bigger patients $(>20 \mathrm{~kg})$ had greater response with AeroEclipse. This new information is important, because AeroEclipse uses less albuterol and less time, with improved efficiency and a lower admission rate than continuous nebulization for the initial aerosol treatment in the ED. Also, patient tolerance of treatment is important in young patients, and the AeroChamber mask used with AeroEclipse seems to be better tolerated. The better efficacy of AeroEclipse may be the result of breath actuation, smaller aerosol particle size, and/or greater patient acceptance. Either way, AeroEclipse appears to be a useful new method of delivering bronchodilator to our young patients, and we have incorporated AeroEclipse into our ED asthma algorithm.

\section{Conclusions}

Albuterol delivery via AeroEclipse did not significantly decrease time in the ED, but it did provide significantly greater improvement in clinical asthma score, better decrease in respiratory rate, and lower hospital admission rate, and was not associated with more adverse effects. Bigger and sicker patients seem to benefit most. This study gives us data to reconsider continuous nebulization in the ED. However, future studies are warranted to compare breath-actuated nebulizers to SVN in subsequent aerosol treatments in the ED, and whether breath-actuated nebulizer can eliminate the use of continuous nebulization.

\section{ACKNOWLEDGMENTS}

We thank Mark Hudes, Children's Oakland Clinical Trials Science Institute, for statistical assistance.

\section{REFERENCES}

1. Rubin BK. Nebulizer therapy for children: the device-patient interface. Respir Care 2002;47(11):1314-1319.

2. Murakami G, Igarashi T, Adachi Y, Matsuno M, Adachi Y, Sawai $\mathrm{M}$, et al. Measurement of bronchial hyperactivity in infants and preschool children using a new method. Ann Allergy 1990;64(4): 383-387.

3. Iles R, Lister P, Edmunds AT. Crying significantly reduces absorption of aerosolized drug in infants. Arch Dis Child 1999;81(2):163165 .

4. Marguet C, Couderc L, Le Roux P, Jeannot E, Lefay V, Mallet E. Inhalation treatments: errors in application and difficulties in acceptance of the devices are frequent in wheezy infants and young children. Pediatr Allergy Immunol 2001;12(4):224-230.

5. Crain EF, Weiss KB, Fagan MJ. Pediatric asthma care in US emergency departments: current practice in the context of the National Institutes of Health guidelines. Arch Pediatr Adolesc Med 1995; 149(8):893-901.

6. Katz RW, Kelly HW, Crowley MR, Grad R, McWilliams BC, Murphy SJ. Safety of continuous nebulized albuterol for bronchospasm in infants and children. Pediatrics 1993;92(2):666-669.

7. Stein J, Levitt MA. A randomized, controlled double-blinded trial of usual-dose versus high-dose albuterol via continuous nebulization in patients with acute bronchospasm. Acad Emerg Med 2003;10(1):3136.

8. Shrestha M, Bidadi K, Gourlay S, Hayes J. Continuous vs intermittent albuterol, at high and low doses, in the treatment of severe acute asthma in adults. Chest 1996;110(1):42-47.

9. Alvine GF, Rodgers P, Fitzsimmons KM, Ahrens RC. Disposable jet nebulizers. How reliable are they? Chest 1992;101(2):316-319.

10. Hess D, Fisher D, Willians P, Pooler S, Kacmarek RM. Medication nebulizer performance: effects of diluent, volume, nebulizer flow and nebulizer brand. Chest 1996;110(2):498-505.

11. American Association for Respiratory Care. Aerosol consensus statement-1991. Respir Care 1991;36(9):916-921.

12. Hess DR, Mitchell JP, Coppolo D, Nagel MW, Archer AD, Blacker R. Effects of nebulizers design on fine particle mass (abstract). Respir Care 1999;44(10):1289. 


\section{Breath-Actuated Nebulizer in Pediatric Asthma}

13. Hess DR, Horney D, Snyder T. Medication delivery: performance of eight small -volume hand held nebulizers: effects of diluent volume, gas flow rate and nebulizer model. Respir Care 1989;34(8):717-723.

14. Hess DR. Nebulizers: principles and performance. Respir Care 2000; 46(6):609-622.

15. Geller DE, Kesser B, Orlando FL. Comparison of drug output from 4 different reservoir-type nebulizers (abstract). Am J Respir Crit Care Med 2001;163(5):444.

16. Rau JL, Ari A, Restrepo RD. Performance comparison of nebulizer designs: constant output, breath-enhanced, and dosimetric. Respir Care 2004;49(2):174-179.

17. Pikarsky R, Farrell T, Acevedo R, Fascia W, Roman C. The delivery time, efficacy, and safety of beta agonist bronchodilator administration with the AERO-ECLIPSE breath actuated nebulizer "BAN" (abstract). Chest 2001;20(4):218S.

18. Leung K, Louca E, Coates A. Comparison of breath-enhanced to breath actuated nebulizers for rate, consistency, and efficiency. Chest 2004;126(5):1619-1627.

19. Anhoj J, Thorsson L, Bisgaard H. Lung deposition of inhaled drugs increases with age. Am J Respir Crit Care Med 2002;162(5):18191822.

20. Kao LC, Warburton D, Platzker AC, Keens TG. Effect of isoproterenol inhalation on airway resistance in chronic bronchopulmonary dysplasia. Pediatrics 1984;73(4):509-514.

21. Gutglass DJ, Hampers L, Roosevelt G, Teoh D, Nimmagadda SR, Krug SE. Undiluted albuterol aerosols in the pediatric emergency department. Pediatrics 2000;105(5):E67.

22. Schuh S, Johnson D, Stephens D, Callahan S, Canny G. Hospitalization patterns in severe acute asthma in children. Pediatr Pulmonol 1997;23(3):184-192.

23. Sabato K, Picchi P, Asselin J, Rutherford M. Use of a clinical asthma score (CAS) as the initial step in an asthma treatment algorithm in a pediatric emergency department (abstract). Respir Care 2002;47(9): 1072.

24. Reisner C, KotchA, Dworkin G. Continuous versus frequent intermittent nebulization of albuterol in acute asthma: a randomized prospective study. Ann Allergy, Asthma Immunol 1995;75(1):41-47.
25. Lin RY, Sauter D, Newman T, Sirleaf J, Walters J, Tavavol M. Continuous versus intermittent albuterol nebulization in the treatment of acute asthma. Ann Emerg Med 1993;22(12):71-77.

26. Papo MC, Frank J, Thompson AE. A prospective, randomized study of continuous versus intermittent nebulized albuterol for severe status asthmaticus in children. Crit Care Med 1993;21(10):14791486.

27. Restrepo RD, Dickson SK, Rau JL, Gardenhire DS. An investigation of nebulized bronchodilator delivery using a pediatric lung model of spontaneous breathing. Respir Care 2006;51(1):56-61.

28. Virant FS. Effective once-daily administration of budesonide inhalation suspension by nebulizer with facemasks or mouthpieces for persistent asthma in infants and young children. Pediatrics 2002; 110(2):460-461.

29. Malone RA, Hollie MC, Glynn- Barnhart A, Nelson HS. Optimal duration of nebulized albuterol therapy. Chest 1993;104(4):11141118.

30. Mellon M, Leflein J, Walton-Bowen K, Cruz-Rivera M, Fitspatrick S, Smith J. Comparable efficacy of administration with face mask or mouthpiece of nebulized budesonide inhalation suspension for infants and young children with persistent asthma. Am J Respir Crit Care Med 2000;162(2 Pt 1):593-598.

31. Lowenthal D, Kattan M. Facemasks versus mouthpieces for aerosol treatment of asthmatic children. Pediatr Pulmonol 1992;14(3):192196.

32. Rubin BK, Fink JB. Aerosol therapy for children. Respir Care Clin N Am 2001;7(2):175-213.

33. Amirav I, Newhouse MT. Aerosol therapy with valved holding chambers in young children: importance of the facemask seal. Pediatrics 2001;108(2):389-394.

34. Geller DE. Kesser B. Blowby vs facemask for nebulized drugs in young children (abstract). J Allergy Clin Immunol 2004;113(2):S32.

35. Everard ML, Clark AR, Milner AD. Drug delivery from nebulisers. Arch Dis Child 1992;67(5):586-591.

36. Rubin BK. Bye bye, blow-by (editorial). Respir Care 2007;52(8): 981.

This article is approved for Continuing Respiratory Care Education credit. For information and to obtain your CRCE

(free to AARC members) visit www.RCJournal.com

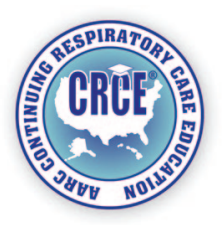

